\title{
AVALIAÇÃO DE UM SISTEMA DE CULTIVO EM ALÉIAS EM UM ARGISSOLO FRANCO-ARENOSO DA REGIÃO AMAZÔNICA(1)
}

\author{
Emanoel Gomes de Moura ${ }^{(2)}$, Antônio José de França Silva ${ }^{(3)}$, \\ Mariléia Barros Furtado ${ }^{(3)} \&$ Alana das Chagas Ferreira Aguiar(4)
}

\begin{abstract}
RESUMO
No trópico úmido, a construção e manutenção da fertilidade dos solos são os maiores desafios dos que se dedicam à implantação de sistemas agrícolas sustentáveis. $O$ objetivo deste estudo foi avaliar um sistema de cultivo em aléias com guandu, associado à adição anual de calcário e de K, em um Argissolo de textura franco-arenosa, a fim de verificar a possibilidade do uso desse sistema como alternativa ao corte e queima na agricultura do trópico úmido. Foram utilizados, como leguminosa, o guandu (Cajanus cajan) e a cultura do milho. Os tratamentos foram os seguintes: $T=$ testemunha, com solo desnudo; $G_{2}, G_{2,5}$ e $G_{3}$, tratamentos com fileiras de guandu nos espaçamentos de $2,2,5$ e $3 \mathrm{~m}$, respectivamente; $\mathrm{G}_{2} \mathrm{~K}, \mathrm{G}_{2,5} \mathrm{~K}$ e $\mathrm{G}_{3} \mathrm{~K}$, tratamentos com guandu nos mesmos espaçamentos mais $\mathrm{K} ; \mathrm{G}_{2} \mathrm{C}, \mathrm{G}_{2,5} \mathrm{C}$ e $\mathrm{G}_{3} \mathrm{C}$, tratamentos com guandu mais calagem; $\mathrm{G}_{2} \mathrm{KC}, \mathrm{G}_{2,5} \mathrm{KC}_{\text {e }} \mathrm{G}_{3} \mathrm{KC}$, tratamentos com guandu mais $\mathrm{K}$ e calagem. A cobertura e o equilíbrio de nutrientes do solo foram os mais importantes fatores que influenciaram a produtividade do milho no sistema de cultivo em aléias com guandu; portanto, eles devem ser considerados como fundamentais para o manejo sustentável dos Argissolos de textura franco-argilosa do trópico úmido.
\end{abstract}

Termos de indexação: Cajanus cajan, milho, produção, adubação potássica, calagem.

\footnotetext{
(1) Recebido para publicação em junho de 2006 e aprovado em junho de 2008.

(2) Professor do Programa de Pós-Graduação em Agroecologia da Universidade Estadual do Maranhão - UEMA. Caixa Postal 3004, CEP 65054-970 São Luís (MA) E-mail: egmoura@elo.com.br

(3) Engenheiros-Agrônomos, Universidade Estadual do Maranhão. E-mail: marileiafurtado@fca.unesp.br

(4) Professora do Centro de Ciências Agrárias e Ambientais, Universidade Federal do Maranhão - UFMA. BR 222, km 04, CEP 65000-000 Chapadinha (MA). E-mail: alanaaguiar@elo.com.br
} 


\title{
SUMMARY: EVALUATION OF AN ALLEY CROPPING SYSTEM UNDER HUMID TROPICAL CONDITIONS OF THE AMAZON REGION
}

\begin{abstract}
In the humid tropics the buildup and maintenance of soil fertility are major challenges in terms of sustainability of agroecosystems. The objective of this study was to evaluate an alley cropping system with pigeon pea (Cajanus cajan) on an Alfisol, with annual application of lime and potassium, with special concern regarding the viability of this system as an alternative to slash and burn practiced in tropical agriculture. Pigeon pea and maize (Zea mays) were planted in the experiment with the following treatments: $T=$ control, with bare soil; G2, G2.5 and G3 = treatments with pigeon pea, spacing between lines of 2, 2.5 and $3 \mathrm{~m}$, respectively; $G 2 K, G 2.5 K$ and $G 3 K=$ pigeon pea, same spacing, plus potassium; $G 2 C$, G2.5C and $G 3 C=$ pigeon pea, same spacing, plus liming; G2KC, G2.5KC and G3KC= pigeon pea, same spacing, plus potassium and liming. Mulch and nutrient balance affected maize yield most in the alley cropping system and must therefore be considered fundamental for the sustainable management of sandy-loamy Alfisols in the humid tropics.
\end{abstract}

Index terms: Cajanus cajan, maize, yield, potassium fertilization, liming.

\section{INTRODUÇÃO}

No trópico úmido, a construção e manutenção da fertilidade dos solos é o maior desafio dos que se dedicam à implantação de sistemas agrícolas sustentáveis, porque se combinam em um mesmo espaço solos altamente intemperizados de baixa capacidade de retenção de cátions com um índice pluviométrico de mais de $2.000 \mathrm{~mm}$ anuais. Nessas condições, o balanço adequado de nutrientes no solo deve levar em conta tanto as altas taxas de remoção de bases do perfil quanto a possibilidade de desordens nutricionais causadas por desequilíbrios entre o excesso de Ca aplicado e a disponibilidade de outros nutrientes.

Como reportou Hartemink (2006), a remoção de bases do perfil em um agroecossistema é governada pela interação entre vários fatores, dentre os quais a CTC e a porosidade do solo, o excesso de precipitação pluvial em relação à evapotranspiração e a capacidade de reciclagem do sistema. Uma fraca interação entre os nutrientes e a matriz do solo, com baixa CTC, pode aumentar a concentração na solução de cátions aplicados; a predominância do Ca, de acordo com Kolahchi \& Jalali (2007), causa a dessorção de outros elementos, especialmente do $\mathrm{K}$, cujas perdas podem atingir até $80 \mathrm{~kg} \mathrm{ha}^{-1}$, como observado por Johnston et al. (1993).

A disponibilidade de micronutrientes no solo está relacionada principalmente ao seu conteúdo total, ao conteúdo do C orgânico e ao pH do solo (Sharma et al., 2004). Deficiências de micronutrientes em solos com baixa acidez estão vastamente documentadas na literatura, como se verifica na revisão de Rashid \& Ryan (2004). Nunes et al. (2004) estudaram as condições que alteram o transporte do $\mathrm{Fe}$ no solo e concluíram que seu fluxo difusivo é altamente dependente da acidez, o que provavelmente deve ser válido também para outros micronutrientes.
Como proposto por Drinkwater \& Snapp (2007), a adoção intencional de processos que enfatizem a construção de reservatórios de nutrientes minerais, acessados por processos microbiologicamente mediados, pode ser uma opção mais adequada para a sustentabilidade dos agroecossistemas do que a saturação por nutrientes da solução do solo, sobretudo no trópico úmido, onde a água excedente aumenta a taxa de remoção dos elementos do perfil. Nessa perspectiva, o cultivo em aléias surgiu na década de 1980 como alternativa promissora para a implantação de agrossistemas familiares, com menor utilização de insumos, por causa de sua eficiência na reciclagem de nutrientes. Duas décadas depois, uma avaliação das experiências, em algumas partes do mundo, mostrou que esse sistema deve ocupar lugares restritos, com situações específicas, onde principalmente não ocorra déficit de água na fase do crescimento das culturas e os riscos da erosão do solo sejam acentuados (Kass et al., 1999; Hauser \& Nolti, 2002; Hauser et al., 2002). Esse sistema de cultivo combina em uma mesma área espécies arbóreas, preferencialmente leguminosas, e culturas anuais, visando reunir em um mesmo espaço e ao mesmo tempo os processos de regeneração da fertilidade do solo e intensificação da ciclagem de nutrientes, as quais são separadas temporariamente na agricultura de derrubada-queima-pousio (Atta-Krah et al., 1989).

De acordo com Mendonça \& Stott (2003), o sucesso de um sistema desse tipo está relacionado com a quantidade e qualidade do material podado das árvores, com a quantidade de nutrientes liberados dos resíduos durante o processo de decomposição e com a quantidade e o tempo de liberação de nutrientes para satisfazer às necessidades das culturas subseqüentes. O guandu (Cajanus cajan (L.) Millsp.) é uma leguminosa forrageira de ciclo anual ou perene, adaptada a ampla faixa de precipitação pluvial, com 
boa resistência à seca e tem seu ótimo em temperaturas mais elevadas (Amabile et al., 2000). Inúmeros autores têm sugerido o guandu como leguminosa para inclusão em sistema de cultivo em aléias, como Salmi et al. (2006), que conseguiram produções entre 4,67 e 5,95 $\mathrm{Mg} \mathrm{ha}^{-1} \mathrm{ano}^{-1}$ de matéria seca, o que incorporou ao sistema $208 \mathrm{~kg} \mathrm{ha}^{-1}$ de $\mathrm{N}$, mais a reciclagem de $8 \mathrm{~kg} \mathrm{ha}^{-1}$ de $\mathrm{Pe} 45 \mathrm{~kg} \mathrm{ha}^{-1}$ de $\mathrm{K}$, por ano.

Além do universo dos indicadores químicos, no trópico úmido, em solos de estrutura frágil, se não houver a proteção da superfície contra o impacto das chuvas, a recompactação da camada arável, como descrito por Busscher (2002), diminui a capacidade de aeração do solo, com efeito negativo sobre o crescimento das plantas suscetíveis ao déficit de $\mathrm{O}_{2}$ nas raízes (Moura et al., 2008).

Os agricultores da região conseguem evitar, em parte, as deficiências de aeração e de nutrientes nas suas áreas de plantio por meio: de uma agricultura itinerante ou sistema de corte e queima, que não inclui a preocupação com a sustentabilidade no uso da terra; do plantio sem preparo, que mantém intacta a estrutura do solo sem quebra da continuidade dos poros, favorecendo a drenagem interna; e do uso da cinza derivada da vegetação natural, como corretivo da acidez e como fonte de nutrientes. No entanto, o aumento da densidade demográfica e do número de agricultores proprietários que derivam dos programas de reforma agrária reforçam a necessidade de substituição do modelo de agricultura itinerante, que não tem garantido nem mesmo a segurança alimentar das famílias. O novo sistema deve levar em conta, além dos princípios de sustentabilidade, as particularidades agroambientais e sociais da região, onde quase a metade da população ainda sobrevive da agricultura, com um dos menores índices de desenvolvimento humano do país (Moura et al., 2008).

O objetivo deste estudo foi avaliar um sistema de cultivo em aléias com guandu, associado à adição anual ou fracionada de calcário e de K, em um Argissolo de textura franco-arenosa, a fim de verificar a possibilidade do uso desse sistema como alternativa ao corte e queima na agricultura itinerante do trópico úmido.

\section{MATERIAL E MÉTODOS}

O experimento foi realizado no Campo Experimental do Núcleo Tecnológico de Engenharia Rural do Curso de Agronomia da Universidade Estadual do Maranhão, no interior da Ilha de São Luís/ MA, situado na região do meio-norte brasileiro, entre a Amazônia úmida e o Nordeste seco, a $44^{\circ} 18^{\prime} \mathrm{W}$ de longitude e $2^{\circ} 30^{\prime} \mathrm{S}$ de latitude. A temperatura média local gira em torno de $26^{\circ} \mathrm{C}$, e as médias máximas e mínimas oscilam entre $28-33$ e $20-23^{\circ} \mathrm{C}$, respectivamente. Tem-se, de modo geral, na região, um período seco de seis a sete meses, dos quais três a quatro muito secos, com menos de $8 \%$ da precipitação pluvial total. No período chuvoso, de cinco a seis meses, pelo menos dois meses podem ser considerados muito chuvosos, com mais de $40 \%$ da precipitação pluvial total e média de $2.300 \mathrm{~mm}$ anuais.

O solo da área é, de acordo com o Sistema Brasileiro de Classificação de Solos, um Argissolo VermelhoAmarelo distrófico arênico textura franco-arenosa (Embrapa, 2006), com as seguintes características químicas: $\mathrm{pH}\left(\mathrm{CaCl}_{2}\right)=4,3 ; \mathrm{P}$ (resina) $=7,0 \mathrm{~g} \mathrm{dm}^{-3}$, $(\mathrm{H}+\mathrm{Al})=33 \mathrm{mmol}_{\mathrm{c}} \mathrm{dm}^{-3} ; \mathrm{K}, \mathrm{Ca}^{2+} \mathrm{e} \mathrm{Mg}^{2+}=1,2,2,0 \mathrm{e}$ $3,0 \mathrm{mmol}_{\mathrm{c}} \mathrm{dm}^{-3}$, respectivamente; $\mathrm{S}=13,2 ; \mathrm{V}(\%)=27$; $\mathrm{CTC}=49,2$; e com as seguintes características físicas: areia fina $=560$, areia grossa $=260$, silte $=80$ e argila $=100 \mathrm{~g} \mathrm{~kg}^{-1}$.

Foi utilizada na implantação das aléias o guandu (Cajanus cajan (L.) Mill sp.), semeado em 1996, nos espaçamentos de 2,0, 2,5 e 3,0 m entre fileiras, com 0,5 m entre plantas. As parcelas, margeadas por duas fileiras de guandu mais uma fileira central, tinham largura de 4, 5 ou $6 \mathrm{~m}$, dependendo das distâncias entre fileiras de guandu. O comprimento era de $21 \mathrm{~m}$, com uma divisão transversal em quatro subparcelas de 5,25 m. O experimento foi delineado em blocos ao acaso, com três repetições dos seguintes tratamentos: solo descoberto (T); $\mathrm{G}_{2}, \mathrm{G}_{2,5}$ e $\mathrm{G}_{3}$, com apenas guandu nos espaçamentos já referidos; $\mathrm{G}_{2} \mathrm{~K}, \mathrm{G}_{2,5} \mathrm{~K}$ e $\mathrm{G}_{3} \mathrm{~K}$, tratamentos com guandu mais $\mathrm{K} ; \mathrm{G}_{2} \mathrm{C}, \mathrm{G}_{2,5} \mathrm{C}$ e $\mathrm{G}_{3} \mathrm{C}$, tratamentos com guandu mais calagem; e $\mathrm{G}_{2} \mathrm{KC}$, $\mathrm{G}_{2,5} \mathrm{KC}$ e $\mathrm{G}_{3} \mathrm{KC}$, tratamentos completos com guandu mais $\mathrm{K}$ e calagem. A cultura do milho (Zea mays) foi semeada no início da estação chuvosa, nos anos de 1997 (I), 1998 (II), 1999 (III) e 2000 (IV), no espaçamento de $80 \mathrm{~cm}$ entre linhas e $20 \mathrm{~cm}$ entre plantas.

A aplicação de calcário foi fracionada em três vezes, nas quais foi aplicado superficialmente o equivalente a 1 t ha-1 de $\mathrm{CaCO}_{3}$, nos anos de 1996, 1997 e 2000. A adubação potássica foi realizada com distribuição a lanço e superficial em uma dose equivalente a $60 \mathrm{~kg} \mathrm{ha}^{-1}$ de $\mathrm{K}_{2} \mathrm{O}$ na forma de $\mathrm{KCl}$, nos quatro anos de cultivo do milho. A cultura do milho recebeu como adubação de plantio 15 e $60 \mathrm{~kg} \mathrm{ha}^{-1}$ de $\mathrm{N}$ e de $\mathrm{P}_{2} \mathrm{O}_{5}$ nas formas de uréia e superfosfato triplo, respectivamente. Na adubação de cobertura foram aplicados $40 \mathrm{~kg}^{-1}$ de $\mathrm{N}$ na forma de uréia, aos 40 dias da emergência.

Logo após o plantio do milho, as plantas de guandu foram cortadas a $40 \mathrm{~cm}$ de altura, e toda a biomassa foi espalhada ao longo das fileiras de milho, de forma que todas as parcelas do mesmo tratamento receberam idêntica quantidade de biomassa. Para avaliar a quantidade de matéria seca do guandu aplicada ao solo, foram separados os ramos correspondentes aos $10 \mathrm{~m}^{2}$ centrais das parcelas. O material foi pesado depois de dividido em colmos e folhas, dos quais foi retirado $1 \mathrm{~kg}$ de cada, e seco em estufa de circulação de ar forçado a $70^{\circ} \mathrm{C}$, para estimativa da matéria seca total aplicada. 
As avaliações das características do solo foram feitas no final dos anos de 1996, 1998 e 2000. Para determinação dos indicadores químicos de fertilidade, foram retiradas amostras na profundidade de 0 a $20 \mathrm{~cm}$; os teores de $\mathrm{Ca}^{2+}, \mathrm{Mg}^{2+}$, $\mathrm{K}$, acidez potencial e matéria orgânica, segundo método da Embrapa (1997). Quanto aos dados da produção do milho, foram contabilizados o número de espigas, o peso de grãos e o peso de 100 grãos, por subparcela.

Os dados foram analisados estatisticamente com auxílio do programa SAEG 9.1 (2007). Os resultados obtidos pelas análises químicas do solo, assim como as produtividades da cultura de milho, foram submetidos à análise de variância, com comparação de médias pelo teste de Tukey a $5 \%$.

\section{RESULTADOS E DISCUSSÃO}

Os resultados das análises químicas do solo realizados no experimento demonstraram alterações em todos os atributos avaliados durante o transcurso do experimento e mostraram que os indicadores químicos da fertilidade foram melhorados por meio da aplicação do calcário, do $\mathrm{K}$ e dos ramos da leguminosa (Quadro 1). Entretanto, nas parcelas com maior aporte de material de cobertura e que receberam calagem, os valores da saturação por bases, no final do experimento, alcançaram valores mais altos que os desejáveis para solos de textura franco-arenosa, como alertaram Sharma et al. (2004), os quais constataram que o teor de $\mathrm{Zn}$ no solo, extraível por DTPA, variou de 1,28 a $0,08 \mathrm{mg} \mathrm{kg}^{-1}$, de acordo com o aumento do $\mathrm{pH}$ e do teor de areia e de Ca.

Os dados de biomassa produzida pelo guandu (Quadro 2) mostram boa relação folha/colmo, em torno de 0,7, comparada a 1,6 para Acacia mangium e Clitoria fairchidiana e 1,3 para Leucaena leococephala, constatadas por Aguiar (2006), quando se considera a cobertura do solo como um dos atributos na escolha da espécie para uso no sistema de cultivo em aléias. Por sua vez, embora os resultados da quantidade de matéria seca adicionada indiquem aumento da produção do guandu com a diminuição do espaçamento, o que se observa na avaliação da produção de biomassa nos espaçamentos de 2 e $3 \mathrm{~m}$ foi que o incremento na produção diminuiu pela metade devido ao aumento do número de plantas. Isso se verifica na comparação das diferenças entre os espaçamentos de 3 com 2,5 (com incremento de $37 \%$ ) e 2,5 com $2 \mathrm{~m}$ (com $16 \%$ ). Uma menor eficiência fotossintética da leguminosa nos espaçamentos menores, em razão do autosombreamento das folhas, pode ter sido a razão dessa diminuição. Isso pode ser importante porque, embora seja desejável maior quantidade de matéria seca, menores espaçamentos entre as árvores aumentam a competição por luz entre as leguminosas e a cultura.

A partir do terceiro ano (1999), houve decréscimo acentuado da produção do guandu, devido ao pouco vigor das brotações e à alta mortalidade das plantas. No momento do corte, no quarto ano (2000), aproximadamente a metade das plantas tinha morrido, o que significa que a sustentabilidade do

\section{Quadro 1. Análise química do solo realizada antes, no final do segundo e do quarto ano do experimento}

\begin{tabular}{|c|c|c|c|c|c|c|c|c|c|c|c|c|c|c|c|c|c|c|}
\hline \multirow{2}{*}{$\begin{array}{c}\text { Nutriente } \\
\text { Ano } \\
\text { Trat. }\end{array}$} & \multicolumn{3}{|c|}{$\mathbf{C a}$} & \multicolumn{3}{|c|}{ Mg } & \multicolumn{3}{|c|}{$\mathbf{K}$} & \multicolumn{3}{|c|}{$\mathbf{H}+\mathbf{A l}$} & \multicolumn{3}{|c|}{ MO } & \multicolumn{3}{|c|}{$\mathbf{V}$} \\
\hline & 1996 & 1998 & 2000 & 1996 & 1998 & 2000 & 1996 & 1998 & 2000 & 1996 & 1998 & 2000 & 1996 & 1998 & 2000 & 1996 & 1998 & 2000 \\
\hline & & & & & & \multicolumn{7}{|c|}{$-\mathrm{cmol}_{\mathrm{c}} \mathrm{dm}^{-3}$} & - & $\mathrm{g} \mathrm{dm}^{-3}$ & - & 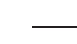 & — \% & - \\
\hline $\mathrm{T}$ & 17 & $13 \mathrm{~b}$ & $10 \mathrm{~b}$ & 8 & $9 \mathrm{bc}$ & $8 c$ & 0,2 & $0,2 \mathrm{~b}$ & $0,1 b$ & 24 & $24 \mathrm{~b}$ & $26 \mathrm{~b}$ & 33 & $26 \mathrm{~b}$ & $28 \mathrm{~b}$ & 52 & $45 \mathrm{c}$ & $36 \mathrm{~d}$ \\
\hline $\mathrm{K}$ & & $14 \mathrm{~b}$ & $16 \mathrm{ab}$ & & $8 b c$ & $11 \mathrm{~b}$ & & $0,6 \mathrm{a}$ & $0,6 \mathrm{a}$ & & $30 \mathrm{a}$ & $30 \mathrm{~b}$ & & $25 \mathrm{~b}$ & $30 \mathrm{~b}$ & & $40 \mathrm{c}$ & $41 d$ \\
\hline $\mathrm{C}$ & & $18 \mathrm{a}$ & $21 \mathrm{a}$ & & $9 \mathrm{bc}$ & $7 \mathrm{c}$ & & $0,1 b$ & $0,1 b$ & & $21 b$ & $18 \mathrm{c}$ & & $22 \mathrm{~b}$ & $28 \mathrm{~b}$ & & $60 \mathrm{a}$ & $65 \mathrm{~b}$ \\
\hline $\mathrm{KC}$ & & $19 \mathrm{a}$ & $22 \mathrm{a}$ & & $9 \mathrm{bc}$ & $7 \mathrm{c}$ & & $0,5 \mathrm{a}$ & $0,1 b$ & & $21 b$ & $19 \mathrm{c}$ & & $27 \mathrm{~b}$ & $27 \mathrm{~b}$ & & $61 \mathrm{a}$ & $65 \mathrm{~b}$ \\
\hline $\mathrm{G}_{2}$ & 16 & $12 \mathrm{~b}$ & $11 \mathrm{~b}$ & 4 & $11 b$ & $12 \mathrm{~b}$ & 0,1 & $0,7 \mathrm{a}$ & $0,1 b$ & 24 & $29 a$ & $28 \mathrm{~b}$ & 28 & $34 \mathrm{a}$ & $32 \mathrm{~b}$ & 45 & $42 \mathrm{~b}$ & $41 d$ \\
\hline $\mathrm{G}_{2} \mathrm{~K}$ & & $13 \mathrm{~b}$ & $12 \mathrm{~b}$ & & $6 \mathrm{c}$ & $3 \mathrm{c}$ & & $0,8 \mathrm{a}$ & $0,9 \mathrm{a}$ & & $24 \mathrm{~b}$ & $22 \mathrm{c}$ & & $36 a$ & $38 \mathrm{a}$ & & $43 \mathrm{~b}$ & $40 \mathrm{~d}$ \\
\hline $\mathrm{G}_{2} \mathrm{C}$ & & $19 a$ & $24 a$ & & $5 c$ & $6 c$ & & $0,1 b$ & $0,2 \mathrm{~b}$ & & $20 \mathrm{~b}$ & $15 \mathrm{c}$ & & $37 \mathrm{a}$ & $39 a$ & & $56 \mathrm{ab}$ & $73 \mathrm{a}$ \\
\hline $\mathrm{G}_{2} \mathrm{KC}$ & & $20 a$ & $23 a$ & & $7 \mathrm{c}$ & $5 c$ & & $0,2 \mathrm{~b}$ & $0,1 b$ & & $21 b$ & $16 \mathrm{c}$ & & $35 a$ & $39 a$ & & $59 \mathrm{a}$ & $67 \mathrm{ab}$ \\
\hline $\mathrm{G}_{2,5}$ & 18 & $12 \mathrm{~b}$ & $14 \mathrm{~b}$ & 2 & $11 b$ & $10 \mathrm{~b}$ & 0,3 & $0,6 \mathrm{a}$ & $0,3 \mathrm{~b}$ & 26 & $29 a$ & $29 \mathrm{~b}$ & 25 & $28 \mathrm{~b}$ & $33 \mathrm{~b}$ & 43 & $41 \mathrm{c}$ & $42 \mathrm{~d}$ \\
\hline $\mathrm{G}_{2,5} \mathrm{~K}$ & & $12 \mathrm{~b}$ & $13 \mathrm{~b}$ & & $8 b c$ & $11 \mathrm{~b}$ & & $0,7 \mathrm{a}$ & $0,6 \mathrm{a}$ & & $23 \mathrm{~b}$ & $28 \mathrm{~b}$ & & $26 \mathrm{~b}$ & $31 \mathrm{~b}$ & & $45 \mathrm{c}$ & $44 \mathrm{~d}$ \\
\hline $\mathrm{G}_{2,5} \mathrm{C}$ & & $19 a$ & $22 \mathrm{a}$ & & $18 \mathrm{a}$ & $20 \mathrm{a}$ & & $0,6 \mathrm{a}$ & $0,2 b$ & & $32 \mathrm{a}$ & $20 \mathrm{c}$ & & $30 \mathrm{ab}$ & $31 \mathrm{~b}$ & & $58 \mathrm{a}$ & $65 \mathrm{~b}$ \\
\hline $\mathrm{G}_{2,5} \mathrm{KC}$ & & $18 \mathrm{a}$ & $23 \mathrm{a}$ & & $5 c$ & $4 \mathrm{c}$ & & $0,7 \mathrm{a}$ & $0,6 a$ & & $23 \mathrm{~b}$ & $18 \mathrm{c}$ & & $32 \mathrm{ab}$ & $30 \mathrm{~b}$ & & $51 b$ & $62 \mathrm{~b}$ \\
\hline $\mathrm{G}_{3}$ & 17 & $14 \mathrm{~b}$ & $20 a$ & 5 & $2 \mathrm{c}$ & $8 \mathrm{c}$ & 0,6 & $0,6 \mathrm{a}$ & $0,4 \mathrm{ab}$ & 34 & $31 \mathrm{a}$ & $32 \mathrm{~b}$ & 25 & $33 \mathrm{ab}$ & $32 \mathrm{~b}$ & 38 & $35 \mathrm{~d}$ & $34 \mathrm{~d}$ \\
\hline $\mathrm{G}_{3} \mathrm{~K}$ & & $13 \mathrm{~b}$ & $12 \mathrm{~b}$ & & $11 b$ & $13 \mathrm{~b}$ & & $0,7 \mathrm{a}$ & $0,5 \mathrm{a}$ & & $36 \mathrm{a}$ & $37 a$ & & $35 a$ & $36 \mathrm{a}$ & & $35 \mathrm{~d}$ & $34 \mathrm{~d}$ \\
\hline $\mathrm{G}_{3} \mathrm{C}$ & & $22 \mathrm{a}$ & $25 \mathrm{a}$ & & $6 \mathrm{c}$ & $5 c$ & & $0,6 \mathrm{a}$ & $0,5 \mathrm{a}$ & & $25 \mathrm{~b}$ & $23 \mathrm{~b}$ & & $30 \mathrm{ab}$ & $37 \mathrm{a}$ & & $54 \mathrm{~b}$ & $59 \mathrm{~b}$ \\
\hline $\mathrm{G}_{3} \mathrm{KC}$ & & $19 a$ & $24 \mathrm{a}$ & & $11 b$ & $7 \mathrm{c}$ & & $0,5 \mathrm{a}$ & $0,5 \mathrm{a}$ & & $30 a$ & $29 \mathrm{~b}$ & & $32 \mathrm{ab}$ & $38 \mathrm{a}$ & & $50 \mathrm{~b}$ & $52 \mathrm{c}$ \\
\hline
\end{tabular}

Médias seguidas das mesmas letras, na coluna, não diferem estatisticamente pelo teste de Tukey a $5 \%$.

T: Solo descoberto; K: solo descoberto, mais potássio, C: solo descoberto, mais calagem; KC: solo descoberto, mais potássio e calagem; $\mathrm{G}_{2}, \mathrm{G}_{2,5}$ e $\mathrm{G}_{3:}$ tratamentos com fileiras de guandu nos espaçamento de dois, dois e meio e três metros; $\mathrm{G}_{2} \mathrm{~K}, \mathrm{G}_{2,5} \mathrm{~K}$ e $\mathrm{G}_{3} \mathrm{~K}$, tratamentos com guandu nos mesmos espaçamentos, mais potássio; $\mathrm{G}_{2} \mathrm{C}, \mathrm{G}_{2,5} \mathrm{C}$ e $\mathrm{G}_{3} \mathrm{C}$, tratamentos com guandu, mais calagem; $\mathrm{G}_{2} \mathrm{KC}, \mathrm{G}_{2,5} \mathrm{KC}$ e $\mathrm{G}_{3} \mathrm{KC}$, tratamentos com guandu mais potássio e calagem. 
sistema, com guandu, dependerá da renovação periódica das sebes. Nas mesmas condições de solo e clima, Ferraz Júnior (2000) comparou o guandu com outras três espécies de leguminosas e observou que Clitoria fairchildiana $\left(12,6 \mathrm{Mg} \mathrm{ha}^{-1}\right)$ e Leucaena leucocephala apresentaram produção ascendente até o quarto ano - época que a produção da primeira foi seis vezes superior à do guandu $\left(2,1 \mathrm{Mg} \mathrm{ha}^{-1}\right)$. No primeiro e no segundo ano, o guandu foi superior às outras leguminosas testadas, totalizando $9,5 \mathrm{Mg} \mathrm{ha}^{-1}$, o que pode ser importante em situações em que se almejam resultados mais imediatos.

Observando os resultados da quantidade de nutrientes reciclados pelo guandu (Quadro 3), percebese relevância maior quanto aos valores do Ca e do N retornados ao solo. Para o $\mathrm{N}$, a eficiência ou o aproveitamento das quantidades recicladas depende do sincronismo entre a liberação e a fase de maior demanda da espécie de interesse econômico. Segundo Buresh \& Tian (1997), essa eficiência é geralmente baixa, ao redor de $22 \%$, significando que somente nos anos 1998, 1999 e 2000 o N adicionado via guandu pode ser considerado relevante. Os resultados da reciclagem de Ca podem ser significativos, levando-se em conta a baixa capacidade de retenção de cátions do solo e que no espaçamento de $2 \mathrm{~m}$ foram restituídos aproximadamente $270 \mathrm{~kg} \mathrm{ha}^{-1} \mathrm{de} \mathrm{Ca}$, nos quatro anos.
A cobertura morta alterou sensivelmente a produção de grãos, principalmente aumentando a massa das espigas, mas as diferenças da produtividade entre os vários espaçamentos do guandu não foram significativas (Quadro 4). Segundo Moura et al. (2008), nesses solos a cobertura favorece a atuação da fauna edáfica, cuja atividade aumenta a capacidade de aeração do solo, que pode ser crítica devido aos períodos de precipitação pluvial intensa, em torno de $2.000 \mathrm{~mm}$ anuais, muito comuns no local. Com a diminuição gradual na produção de biomassa do guandu, o sistema não suportou a sustentabilidade da produção do milho, ainda que na soma dos quatro anos a produção das parcelas cobertas tenha sido superior a $20 \%$, comparadas com as parcelas que não receberam a cobertura. De maneira similar, na mesma região, Ferraz Júnior (2000) demonstrou que as espécies arbóreas, como C. fairchildiana, podem, em longo prazo, ser alternativas mais adequadas quanto às quantidades e à estabilidade na produção de biomassa de baixa qualidade. A melhor qualidade da biomassa nem sempre deve ser o critério para escolha de uma espécie para uso no cultivo em aléias, onde o efeito do resíduo está mais condicionado à proteção da superfície do solo do que ao fornecimento de nutrientes. Algo similar tem sido relatado por Tian et al. (1997), em que as melhores respostas dos cultivos se

Quadro 2. Quantidade de matéria seca produzida pelo guandu e adicionada ao solo nos três espaçamentos

\begin{tabular}{|c|c|c|c|c|c|c|c|c|c|c|c|c|}
\hline \multirow{2}{*}{ Espaçamento } & \multicolumn{4}{|c|}{ Folha } & \multicolumn{4}{|c|}{ Colmo } & \multicolumn{4}{|c|}{ Total } \\
\hline & 1997 & 1998 & 1999 & 2000 & 1997 & 1998 & 1999 & 2000 & 1997 & 1998 & 1999 & 2000 \\
\hline $\mathrm{m}$ & \multicolumn{12}{|c|}{$-\mathrm{Mg} \mathrm{ha}^{-1}$} \\
\hline 3,0 & $0,36 \mathrm{a}$ & $2,6 \mathrm{~b}$ & $1,1 \mathrm{~b}$ & $0,5 b$ & $0,52 b$ & $3,6 \mathrm{~b}$ & $1,5 b$ & $0,7 \mathrm{a}$ & $0,9 \mathrm{~b}$ & $6,2 \mathrm{~b}$ & $2,6 \mathrm{~b}$ & $1,2 \mathrm{~b}$ \\
\hline 2,5 & $0,49 a$ & $3,4 \mathrm{a}$ & $1,5 \mathrm{a}$ & $0,7 \mathrm{ab}$ & $0,71 \mathrm{a}$ & $4,7 \mathrm{a}$ & $1,9 a b$ & $0,9 \mathrm{a}$ & $1,2 \mathrm{a}$ & $8,1 \mathrm{a}$ & $3,4 \mathrm{ab}$ & $1,6 \mathrm{ab}$ \\
\hline 2,0 & $0,61 \mathrm{a}$ & $3,9 a$ & $1,7 \mathrm{a}$ & $0,8 \mathrm{a}$ & $0,73 a$ & $5,5 \mathrm{a}$ & $2,2 \mathrm{a}$ & $1,1 \mathrm{a}$ & $1,4 \mathrm{a}$ & $9,4 \mathrm{a}$ & $3,9 \mathrm{a}$ & $1,9 \mathrm{a}$ \\
\hline CV (\%) & 15,1 & 12,3 & 17,2 & 10,6 & 12,5 & 16,9 & 14,2 & 13,2 & 20 & 14,4 & 17,1 & 16,4 \\
\hline
\end{tabular}

Médias seguidas das mesmas letras, na coluna, não diferem estatisticamente pelo teste de Tukey a $5 \%$.

Quadro 3. Quantidade de nutrientes adicionados ao solo pelo guandu nos três espaçamentos

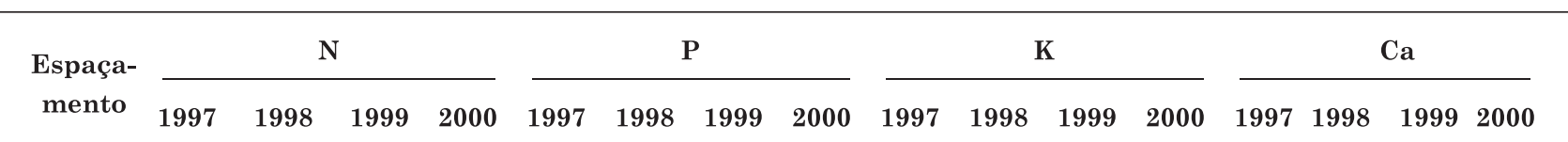

\begin{tabular}{|c|c|c|c|c|c|c|c|c|c|c|c|c|c|c|c|c|}
\hline \multirow{2}{*}{$\begin{array}{c}\mathrm{m} \\
3,0\end{array}$} & \multicolumn{16}{|c|}{$\mathrm{kg} \mathrm{ha}^{-1}$} \\
\hline & $28 \mathrm{c}$ & $198 b$ & $82 \mathrm{c}$ & $35 c$ & $1,8 \mathrm{~b}$ & $12 \mathrm{c}$ & $3,0 \mathrm{c}$ & $2,5 b$ & $4,5 b$ & $30 \mathrm{~b}$ & $13 \mathrm{c}$ & $6,0 \mathrm{c}$ & $14 \mathrm{~b}$ & $95 \mathrm{c}$ & $39 c$ & $18 \mathrm{~b}$ \\
\hline 2,5 & $38 \mathrm{~b}$ & $240 \mathrm{ab}$ & $114 \mathrm{~b}$ & $45 \mathrm{~b}$ & $2,4 \mathrm{a}$ & $16 \mathrm{~b}$ & $6,2 \mathrm{~b}$ & $3,0 \mathrm{~b}$ & $6,0 \mathrm{a}$ & $40 a$ & $16 \mathrm{~b}$ & $8,0 \mathrm{~b}$ & $17 \mathrm{a}$ & $120 \mathrm{~b}$ & $51 b$ & $25 \mathrm{ab}$ \\
\hline 2,0 & $45 \mathrm{a}$ & $275 \mathrm{a}$ & $121 \mathrm{a}$ & $61 \mathrm{a}$ & $2,8 \mathrm{a}$ & $18 \mathrm{a}$ & $8,1 \mathrm{a}$ & $4,1 \mathrm{a}$ & $7,0 \mathrm{a}$ & $46 \mathrm{a}$ & $21 a$ & $11 \mathrm{a}$ & $21 a$ & $161 \mathrm{a}$ & $60 a$ & $30 \mathrm{a}$ \\
\hline CV (\%) & 12,1 & 14,5 & 15,3 & 10,1 & 15,3 & 17,8 & 10,4 & 11,7 & 18,1 & 13,5 & 17,6 & 12,3 & 20 & 17,5 & 14,3 & 12,3 \\
\hline
\end{tabular}

Médias seguidas das mesmas letras, na coluna, não diferem estatisticamente pelo teste de Tukey a $5 \%$. 
alcançam com a adição da biomassa de decomposição mais lenta.

A importância da cobertura para a sustentabilidade da produção, neste experimento, pode também ser avaliada quando se observa a redução da produção na área descoberta, tanto que no quarto ano (2000) a produção desse tratamento atingiu pouco mais da metade das áreas que receberam cobertura.

No segundo ano, a adubação potássica e a calagem juntas (Quadro 5) aumentaram significativamente os indicadores de produtividade do milho, principalmente a massa de espigas e de 100 grãos, refletindo o efeito da correção dos inadequados níveis de acidez, de saturação por bases e de K, originais do solo. A partir do terceiro ano, as plantas das parcelas apenas calcariadas produziram espigas de menor massa, provavelmente por causa da diminuição da disponibilidade dos micronutrientes, sobretudo do Zn, com o aumento do $\mathrm{pH}$ e dos teores de $\mathrm{Ca}$, acima do que seria adequado em solos de textura franco-arenosa. Desordem nutricional nessas condições foi também observada por Brennan et al. (2005).
No quarto ano (2000), as plantas das parcelas calcariadas apresentaram sintomas claros de deficiência nutricional com a diminuição das distâncias entre os internós e com o amarelecimento das folhas, o que refletiu de maneira negativa no peso das espigas e na produção de grãos, principalmente nas parcelas sem K. Esses resultados mostram que, para solos com baixa capacidade de tamponamento, os níveis de saturação por bases mais adequados e, por conseqüência, a quantidade de calcário a ser aplicada divergem daqueles apresentados em tabelas de recomendações para outras regiões, como a de Raij et al. (1997) e da Comissão de Fertilidade do Solo do Estado de Minas Gerais (CFSEMG), que recomendam para o milho a saturação de $70 \%$. Nas condições deste experimento, maiores produtividades foram alcançadas com saturação em torno de $50 \%$.

\section{CONCLUSÕES}

1. A cobertura e o equilíbrio de nutrientes do solo foram os mais importantes fatores que influenciaram

Quadro 4. Efeito dos espaçamentos entre fileiras de guandu sobre indicadorse de produção do milho

\begin{tabular}{|c|c|c|c|c|c|c|c|c|c|c|c|c|}
\hline \multirow{2}{*}{ Tratamento } & \multicolumn{4}{|c|}{ Espiga } & \multicolumn{4}{|c|}{ Peso de 100 grãos } & \multicolumn{4}{|c|}{ Peso de grãos } \\
\hline & 1997 & 1998 & 1999 & 2000 & 1997 & 1998 & 1999 & 2000 & 1997 & 1998 & 1999 & 2000 \\
\hline & \multicolumn{4}{|c|}{$-\mathrm{Mg} \mathrm{ha}^{-1}$} & \multicolumn{4}{|c|}{$-\mathrm{g}$} & \multicolumn{4}{|c|}{$-\mathrm{Mg} \mathrm{ha}^{-1}$} \\
\hline $\mathrm{T}$ & $5,3 \mathrm{~b}$ & $6,6 \mathrm{~b}$ & $4,9 \mathrm{a}$ & $2,3 \mathrm{~b}$ & $31,1 \mathrm{a}$ & $13,5 b$ & $26,6^{\text {a }}$ & $24,0 \mathrm{a}$ & $4,6 \mathrm{~b}$ & $3,2 \mathrm{~b}$ & $4,2 \mathrm{a}$ & $1,8 \mathrm{~b}$ \\
\hline $\mathrm{G}_{2}$ & $6,0 \mathrm{ab}$ & $7,8 \mathrm{a}$ & $5,7 \mathrm{a}$ & $3,8 \mathrm{ab}$ & $30,4 \mathrm{a}$ & $16,6 a b$ & $26,9 \mathrm{a}$ & $25,3 \mathrm{a}$ & $5,2 \mathrm{ab}$ & $4,3 \mathrm{a}$ & $5,0 \mathrm{a}$ & $3,5 \mathrm{ab}$ \\
\hline $\mathrm{G}_{2,5}$ & $6,2 \mathrm{ab}$ & $7,0 \mathrm{ab}$ & $4,8 \mathrm{a}$ & $3,9 \mathrm{ab}$ & $30,2 \mathrm{a}$ & $16,9 \mathrm{a}$ & $27,4 \mathrm{a}$ & $26,2 \mathrm{a}$ & $5,4 \mathrm{ab}$ & $3,6 \mathrm{ab}$ & $4,0 \mathrm{a}$ & $3,3 \mathrm{a}$ \\
\hline $\mathrm{G}_{3}$ & $6,5 \mathrm{a}$ & $7,4 \mathrm{ab}$ & $4,5 \mathrm{a}$ & $4,4 \mathrm{a}$ & $31,1 \mathrm{a}$ & $15,1 \mathrm{ab}$ & $26,9 \mathrm{a}$ & $25,0 \mathrm{a}$ & $5,6 a$ & $3,8 \mathrm{ab}$ & $3,8 \mathrm{a}$ & $3,6 \mathrm{a}$ \\
\hline CV (\%) & 20 & 6,6 & 12,1 & 16,4 & 7 & 7,2 & 4,1 & 16,4 & 20 & 7,1 & 11,4 & 16,4 \\
\hline
\end{tabular}

T: solo descoberto; $\mathrm{G}_{2}, \mathrm{G}_{2,5}$ e $\mathrm{G}_{3}$. tratamentos com fileiras de guandu nos espaçamento de dois, dois e meio e três metros. Médias seguidas das mesmas letras, nas colunas, não diferem estatisticamente pelo teste de Tukey a $5 \%$.

Quadro 5. Efeito da adubação potássica e da calagem sobre os parâmetros da produção do milho

\begin{tabular}{|c|c|c|c|c|c|c|c|c|c|c|c|c|}
\hline \multirow{2}{*}{ Tratamento } & \multicolumn{4}{|c|}{ Espiga } & \multicolumn{4}{|c|}{ Peso de 100 grãos } & \multicolumn{4}{|c|}{ Peso de grãos } \\
\hline & 1997 & 1998 & 1999 & 2000 & 1997 & 1998 & 1999 & 2000 & 1997 & 1998 & 1999 & 2000 \\
\hline & \multicolumn{4}{|c|}{$-\mathrm{Mg} \mathrm{ha}^{-1}$} & \multicolumn{4}{|c|}{$-\mathrm{g}$} & \multicolumn{4}{|c|}{$\mathrm{Mg} \mathrm{ha}^{-1}$} \\
\hline $\mathrm{T}$ & $6,2 \mathrm{a}$ & $6,6 \mathrm{~b}$ & $4,7 \mathrm{a}$ & $3,3 \mathrm{a}$ & $31,1 \mathrm{a}$ & $13,5 \mathrm{c}$ & $26,9 \mathrm{a}$ & $23,1 \mathrm{a}$ & $5,4 \mathrm{a}$ & $3,2 \mathrm{~b}$ & $2,9 \mathrm{a}$ & $2,7 \mathrm{ab}$ \\
\hline $\mathrm{K}$ & $5,9 \mathrm{a}$ & $6,8 \mathrm{~b}$ & $5,0 \mathrm{a}$ & $3,9 \mathrm{a}$ & $30,7 \mathrm{a}$ & $14,8 \mathrm{~b}$ & $27,0 \mathrm{a}$ & $26,0 \mathrm{a}$ & $5,1 \mathrm{a}$ & $3,5 \mathrm{~b}$ & $4,2 \mathrm{a}$ & $3,2 \mathrm{a}$ \\
\hline $\mathrm{C}$ & $5,7 \mathrm{a}$ & $6,9 \mathrm{~b}$ & $3,9 \mathrm{~b}$ & $2,8 \mathrm{~b}$ & $30,3 a$ & $16,9 \mathrm{ab}$ & $26,8 \mathrm{a}$ & $25,1 \mathrm{a}$ & $4,9 \mathrm{a}$ & $3,5 \mathrm{~b}$ & $3,1 b$ & $2,3 \mathrm{~b}$ \\
\hline $\mathrm{KC}$ & $6,3 \mathrm{a}$ & $8,1 \mathrm{a}$ & $5,1 \mathrm{a}$ & $3,7 \mathrm{a}$ & $30,6 \mathrm{a}$ & $18,6 \mathrm{a}$ & $27,0 \mathrm{a}$ & $26,4 \mathrm{a}$ & $5,5 \mathrm{a}$ & $4,3 \mathrm{a}$ & $4,2 \mathrm{a}$ & $3,0 \mathrm{ab}$ \\
\hline $\mathrm{CV}(\%)$ & 20 & 6,6 & 8,7 & 16,4 & 7,0 & 7,1 & 1,2 & 16,4 & 20 & 7,2 & 8,4 & 16,4 \\
\hline
\end{tabular}

T: solo descoberto; K: solo descoberto, mais potássio, C: solo descoberto, mais calagem; KC: solo descoberto, mais potássio e calagem. Médias seguidas das mesmas letras, nas colunas, não diferem estatisticamente pelo teste de Tukey a $5 \%$. 
a produtividade do milho no sistema de cultivo em aléias com guandu e, portanto, devem ser considerados como fundamentais para o manejo sustentável dos Argissolos de textura franco-argilosa do trópico úmido.

2. O cultivo em aléias com guandu pode ser uma boa alternativa para o manejo de solos no trópico úmido, devido à sua eficiência na reciclagem do $\mathrm{N}$ e do Ca e por sua capacidade de fornecer biomassa para cobertura do solo logo a partir do segundo ano de plantio.

3. A recomendação das quantidades e dos intervalos de aplicação de calcário e do K nessas condições deve ser aprimorada em trabalhos futuros, que levem em conta o nível de saturação por bases do solo, bem como a capacidade de reciclagem do sistema.

\section{LITERATURA CITADA}

AGUIAR, A.C.F. Sustentabilidade do sistema plantio direto em Argissolo no trópico úmido. Botucatu, Universidade Estadual Paulista, 2006. 57p. (Tese de Doutorado)

ALBUQUERQUE, J.M. Níveis de preparo e de cobertura entre aléias de guandu com milho, como alternativas de melhoramento da qualidade física e do uso intensivo de um Argissolo da Formação Itapecuru-MA. São Luís, Universidade Estadual do Maranhão, 1999. 66p. (Tese de Mestrado)

AMABILE, R.F.; FANCELLI, A.L. \& CARVALHO, A.M. Comportamento de espécies de adubos verdes em diferentes épocas de semeadura e espaçamentos na região dos Cerrados. Pesq. Agropec. Bras., 35:47-54, 2000.

ATTA-KRAH, A.N. Alley farming with leucaena: Effects of short grazed fallows on soil fertility and crop yields. Exper. Agric., 20:1-10, 1989.

BRENNAN, R.F.; BOLLAND, M.D.A. \& BELL, R.W. Increased risk of zinc deficiency in wheat on soils limed to correct soil acidity. Aust. J. Soil Res., 43:647-657, 2005.

BURESH, R.J. \& TIAN, G. Soil improvement by trees in subSaharan Africa. Agrofo. Sys. 38:51-76, 1997.

BUSSCHER, W.J.; BAUER, P.J. \& FREDERICK, J.R. Recompaction of the coastal loamy sand after deep tillage as a the function of subsequent cumulative rainfall. Soil Till. Res., 68:49-57, 2002.

DRINKWATER, L.E. \& SNAPP, S.S. Nutrients in agroecosystems: Rethinking the management paradigm. Adv.Agron., 92:163-186, 2007.

EMPRESA BRASILEIRA DE PESQUISA AGROPECUÁRIA EMBRAPA. Serviço Nacional de Levantamento e Conservação de Solos. Manual de métodos de análise de solo. 2.ed. Rio de Janeiro, 1997. 212p.
EMPRESA BRASILEIRA DE PESQUISA AGROPECUÁRIA EMBRAPA. Serviço Nacional de Levantamento e Conservação de Solos. Sistema brasileiro de classificação de solos. 2.ed. Rio de Janeiro, 2006. 306p.

FERRAZ JUNIOR, A.S.L. Arroz de sequeiro em aléias de leguminosas sobre solo de baixa fertilidade natural. Seropédica, Universidade Federal Rural do Rio de Janeiro, 2000. 126p. (Tese de Doutorado)

HAUSER, S. \& NOLTI, C. Biomass production and N fixation of five Mucuna pruriens varieties and their effect on maize yields in the forest zone of Cameroon. J. Plant Nut. Soil Sci., 165:101-109, 2002.

HAUSER, S.; HENROT, J. \& HAUSER, A. Maize yields in mulched and burned Mucuna pruriens var. utilis and Pueraria phaseoloides relay fallow systems in Southern Cameroon. Biol. Agric Hortic., 20:243-256, 2002.

HARTEMINK, A.E. Assessing soil fertility decline in the tropics using soil chemical data. Adv. Agron., 89:179-225, 2006.

JOHNSTON, A.E.; GOULDING, K.W.T. \& MERCER, E. Potassium leaching from a sandy soil. Bern, International Potash Institute, 1993. (Subject 12)

KASS, D.C.L.; THURSTON, H.D. \& SCHLATHER, K. Sustainable mulch-based cropping systems with trees. In: BUCK, L.E.; LASSOIE, J.P. \& FERNANDES, E.C.M. eds. Agroforestry in sustainable agricultural systems. New York, CRC Press, 1999. p.361-379.

KOLAHCHI, Z. \& JALALI, M. Effect of water quality on the leaching of potassium from 16 sandy soils. J. Arid Environ., 68:624-639, 2007.

MENDONÇA, E.S. \& STOTT, D.E. Characteristics and decomposition rates of pruning residues from a shaded coffee system in Southeastern Brazil. Agrofor. Syst., 57:117-125, 2003.

MOURA, E.G. Agroambientes de transição avaliados numa perspectiva da agricultura familiar. In: MOURA, E.G., org. Agroambientes de transição entre o trópico úmido e o semi-árido do Brasil: Atributos; alternativas; uso na produção familiar. 2.ed. São Luís, Universidade Estadual do Maranhão, 2006. p.15-51.

MOURA, E.G.; ALBUQUERQUE, J.M. \& AGUIAR, A.C.F. Growth and productivity of corn as affected by mulching and tillage in alley cropping systems. Sci. Agric. 65:204208,2008

NUNES, F.N.; NOVAIS, R.F.; SILVA, I.R.; GEBRIM, F.O. \& JOSE, J.F.B.S. Diffusive flux of iron in soils influenced by phosphorus rates and levels of acidity and moisture. R. Bras. Ci. Solo, 28:423-429, 2004.

RAIJ, B.van; CANTARELA, H.; QUAGGIO, J.A. \& FURLANI, A.M.C. Recomendações de adubação e calagem para o Estado de São Paulo. Campinas, Instituto Agronômico, 1997. 285p. (Boletim técnico, 100)

RASHID, A. \& RYAN, J. Micronutrient constraints to crop production in soils with Mediterranean-type characteristics: A review. J. Plant Nutr., 27:959-975, 2004. 
SISTEMA PARA ANÁLISES ESTATÍSTICAS - SAEG. Versão 9.1. Viçosa, MG, Fundação Arthur Bernardes/ Universidade Federal de Viçosa, 2007.

SALMI, G.P.; SALMI, A.P. \& ABBOUD, A.C.S. Decomposition dynamics and nutrient release by pigeon pea genotypes under alley cropping. Pesq. Agropec. Bras., 41:673-678, 2006.
SHARMA, B.D.; ARORA, H.; KUMAR, R. \& NAYYAR, V.K. Relationships between soil characteristics and total and DTPA-extractable micronutrients in Inceptisols of Punjab. Comm. Soil Sci. Plant Anal., 35:799-818, 2004.

TIAN, G.; KANG, B.T. \& BRUSSAARD, L. Effect of mulch quality on earthworm activity and nutrient supply in the humid tropics. Soil Biol. Biochem., 29:369-373, 1997. 